\title{
Cirurgias Colorretais no Hospital Universitário da Universidade Federal de Sergipe: Três Anos de Criação do Serviço de Coloproctologia (Série Histórica)
}

\author{
Colorrectal Surgery at the Federal University Hospital of Sergipe: An Overview \\ of Three Years After the Creation of the Colorretal Group
}

\author{
JUVENALDA ROCHATORRES NETO'; MAGNO CÉZAR AMARALDE SOUZAJÚNIOR²; RODRIGO ROCHA \\ SANTIAGO ${ }^{3}$;ANACAROLINALISBOAPRUDENTE ${ }^{4}$
}

\begin{abstract}
${ }^{1}$ Professor, Doutor e Chefe do Serviço de Coloproctologia $;{ }^{2}$ Médico residente em cirurgia geral; ${ }^{3}$ Médico assistente titular do Serviço de Coloproctologia: ${ }^{4}$ Médica residente em coloproctologia. Hospital Universitário da Universidade Federal de Sergipe - BRASIL
\end{abstract}

TORRES NETO JR; SOUZA JR MCA; SANTIAGO RR; PRUDENTE ACL. Cirurgias colorretais no Hospital Universitário da Universidade Federal de Sergipe: três anos de criação do serviço de coloproctologia (série histórica). Rev bras Coloproct, 2008;28(1): 077-083.

RESUMO: Estudamos cirurgias colorretais do SC-HU/UFS (Serviço de Coloproctologia do Hospital Universitário da Universidade Federal de Sergipe) realizadas de janeiro de 2004 a julho de 2006, série histórica da criação da residência médica em coloproctologia. De setenta pacientes, $53(75,7 \%$ ) eram do gênero masculino, 17(24,3\%) feminino; idade variou de 19 a 85 anos com média de 52 anos. Os setenta pacientes foram submetidos a 102 procedimentos, com média de 1,4 cirurgias/paciente, $\mathbf{2 9}(\mathbf{2 8 , 4 \%})$ reoperações. Dezenove $(\mathbf{1 8 , 6 \%})$ foram reconstituições de trânsito intestinal, $15(14,8 \%)$ retossigmoidectomias, $\mathbf{1 1}(10,8 \%)$ colectomias totais, $9(8,8 \%)$ colectomias direitas, $6(5,8 \%)$ amputações abdomino-perineais, $3(2,9 \%)$ proctocolectomias, $\mathbf{2}(1,9 \%)$ colectomias esquerdas. Dezoito $(17,6 \%)$ cirurgias indicadas por neoplasias de cólon, $8(7,8 \%)$ do reto e $1(0,9 \%)$ do canal anal; 10(9,9\%) foram megacólon; 10(9,9\%) colostomias prévias, 5(5,9\%) Doença de Crohn, 5(4,9\%) DDC, 3(2,9\%) RCUI. Quarenta e oito $(47,1 \%)$ cirurgias tiveram complicações cirúrgica: $32(31,4 \%)$ complicações da ferida operatória, $13(12,7 \%)$ abscessos abdominais, 11(10,8\%) fístulas, 7(6,9\%) deiscências, etc. Índice de infecção de ferida foi 26,5\%. Cinqüenta e cinco pacientes $(53,9 \%)$ foram submetidos à anastomose, 32(58,2\%) manuais e 23(41,8\%) mecânicas. Deiscência de anastomose em 7(12,7\%) cirurgias: $1(3,1 \%)$ deiscência em anastomose manual e 6(26,1\%), em mecânica. Óbito em 11(15,7\%) pacientes. Avaliamos principais dados do trabalho objetivando definir metas, elaborar e melhorar protocolos vigentes objetivando otimizar o programa de residência médica.

Descritores: Cirurgia colorretal, complicações pós-operatórias, Neoplasias Colorretais, Colite Ulcerativa, Doenças de Chagas.

\section{INTRODUÇÃOO}

Apesar de o Serviço de Coloproctologia do Hospital Universitário de Sergipe (SC-HU/UFS) funcionar há muitos anos, inserido no Serviço de Cirurgia Geral, só recentemente ele foi estruturado, obtendo reconhecimento da Sociedade Brasileira de Coloproctologia (SBCP) e da Comissão Nacional de Residência Médica, como serviço independente.
As cirurgias abdominais colorretais são realizadas no trauma e diante de patologias como câncer colorretal, doença diverticular dos cólons, megacólon chagásico, hemorragia digestiva baixa e doença inflamatória intestinal.

É imprescindível que os serviços médicos organizem-se baseados em evidências, transformando uma medicina de ações subjetivas, numa medicina de condutas sólidas e objetiva. Assim, é possível mensurar

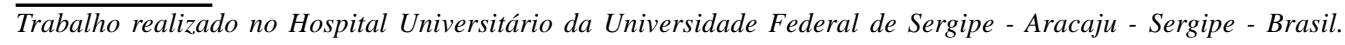


e comparar resultados criteriosamente. O processo de formação médica está intimamente ligado à medicina baseada em evidências ${ }^{(1)}$.

Esse trabalho tem o propósito de analisar os dados levantados sobre todas as cirurgias colorretais desde a estruturação do serviço, publicando-os como uma série histórica, para subsidiar elaboração e/ou complementação dos diversos protocolos, enfatizando a qualidade dos serviços prestados enquanto instituição formadora de especialistas.

\section{CASUÍSTICA E MÉTODOS}

Foram coletadas de forma retrospectiva, todas as cirurgias abdominais realizadas no SC-HU/UFS entre janeiro de 2004 até julho de 2006.

Inicialmente, era preenchida uma lista padrão de coleta baseada no livro de registro de sala associado aos prontuários de ambulatório. Foram registrados: identificação, registro hospitalar, datas de admissão, cirurgia e de alta, data de nascimento, gênero, procedência, diagnóstico inicial, co-morbidades, profilaxia da TVP / TEP, uso de antibiótico, classificação de risco anestésico (ASA), cirurgia realizada, classificação da cirurgia quanto ao risco de infecção, tipo de anestesia, tempo cirúrgico, confecção de ostomias, anastomose mecânica ou manual, complicações relacionadas à ferida ou à anastomose (deiscência/abscesso/fístula/obstrução), anátomo-patológico e óbito.

Todos estes dados eram dispostos em fichas pré-elaboradas em software Word ${ }^{\circledR}$ (Apêndice A) e, após, trabalhados sob a forma de planilhas/banco de dados no software Excel $^{\circledR}$. Não foi necessária aplicação de testes estatísticos. A análise descritiva foi realizada através do sistema de filtro do próprio programa.

\section{RESULTADOS}

No período de janeiro de 2004 a julho de 2006, 70 pacientes foram submetidos a 102 procedimentos cirúrgicos (média de 1,4 cirurgias/paciente). Dezesseis $(22,8 \%)$ pacientes foram submetidos a mais de um procedimento cirúrgico, num total de $27(26,4 \%)$ cirurgias - tabela (1). Cinquienta e três $(75,7 \%)$ pacientes eram do gênero masculino e 17 (24,3\%), feminino. A idade variou de 19 a 85 anos, com média de 52 anos. Sessenta e um $(87,2 \%)$ eram procedentes do estado de Sergipe, sendo $35(50 \%)$ do interior e $26(37,2 \%)$ da capital. Nove $(12,8 \%)$ procediam do estado da Bahia.
Os pacientes foram submetidos a diversos procedimentos, conforme tabela (2).

Os pacientes tiveram como diagnóstico inicial neoplasia de cólon, reto e ânus em $26,4 \%$ dos casos, megacólon em 9,9\% e, DII perfazendo 8,8\% do total tabela (3).

Sessenta e um $(59,8 \%)$ pacientes foram classificados como ASA I, 34 (33,3\%) ASA II, 7 (6,9\%) ASA III.

Tabela 1 - Número de cirurgias por paciente.

\begin{tabular}{ccc}
\hline $\mathbf{N}^{\mathbf{0}}$ & pacientes & Número de cirurgias por paciente \\
\hline 9 & $(5,7 \%)$ & 2 \\
4 & $(2,9 \%)$ & 3 \\
2 & $(2,9 \%)$ & 4 \\
1 & $(1,4 \%)$ & 5 \\
\hline
\end{tabular}

Tabela 2 - Procedimentos cirúrgicos realizados.

\begin{tabular}{lrr}
\hline Tipos de cirurgias & & \\
\hline Drenagem e lavagem de cavidade & & \\
Abdominal, enterectomias, colostomias & 37 & $(36,3 \%)$ \\
Reconstituições de trânsito intestinal & 19 & $(18,6 \%)$ \\
Retossigmoidectomias & 15 & $(14,8 \%)$ \\
Colectomias direitas & 11 & $(10,8 \%)$ \\
Colectomias totais & 9 & $(8,8 \%)$ \\
Amputações abdomino-perineais & 6 & $(5,8 \%)$ \\
Proctocolectomias & 3 & $(2,9 \%)$ \\
Colectomias esquerdas & 2 & $(1,9 \%)$ \\
\hline
\end{tabular}

Tabela 3 - Diagnóstico inicial.

\begin{tabular}{lrc}
\hline Diagnóstico inicial & & \\
\hline Neoplasia de cólon & 18 & $(17,6 \%)$ \\
Neoplasia do reto & 8 & $(7,8 \%)$ \\
Neoplasia do canal anal & 1 & $(0,9 \%)$ \\
Megacólon & 10 & $(9,9 \%)$ \\
Colostomias prévias & 10 & $(9,9 \%)$ \\
DDC & 5 & $(5,9 \%)$ \\
Doença de Crohn & 5 & $(5,9 \%)$ \\
RCUI & 3 & $(2,9 \%)$ \\
Deiscências & 10 & $(9,9 \%)$ \\
Abscessos abdominais & 8 & $(7,8 \%)$ \\
Fístulas & 5 & $(4,9 \%)$ \\
\hline
\end{tabular}


Rev bras Coloproct

Janeiro/Março, 2008
Cirurgias Colorretais no Hospital Universitário da Universidade Federal de Sergipe: Três Anos da Criação do Serviço de Coloproctologia (Série Histórica) Juvenal da Rocha Torres Neto e Cols.
Vol. 28

\section{APÊNDICE A}

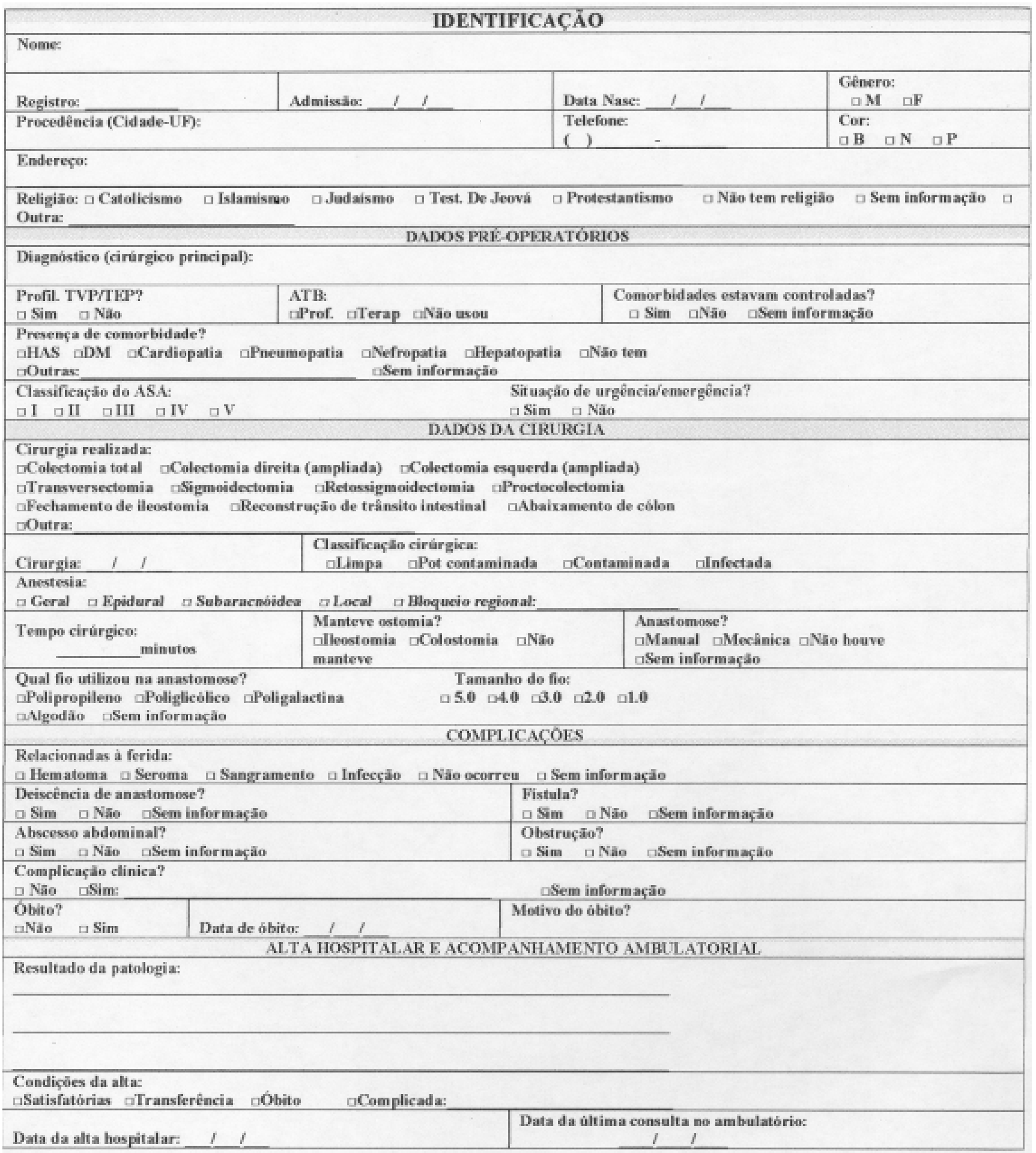

Figura 1 - Ficha utilizada para coleta de dados de pacientes cujos prontuários foram revisados. 
Setenta e uma $(69,6 \%)$ cirurgias foram eletivas e $31(30,4 \%)$ foram urgências.

O protocolo do serviço para preparo de cólon é Manitol ${ }^{\circledR}$ a $10 \%$ e fosfato de Sódio - Fleet Enema ${ }^{\circledR}$. Em $54(52,9 \%)$ procedimentos foi usado esse protocolo, $7(6,9 \%)$ usaram somente Manitol ${ }^{\circledR}$ oral, $5(4,9 \%)$ usaram Fleet Enema ${ }^{\circledR}$ e 36 (35,3\%) não foram submetidos a preparo. Os procedimentos que não usaram preparo de cólon eram cirurgias de urgência.

Quando analisamos a incidência de deiscência e infecção da ferida operatória (FO) com relação à presença ou não de preparo, temos dados da tabela (4).

Oitenta e dois $(80,4 \%)$ pacientes foram submetidos à profilaxia TVP/TEP.

Sessenta e cinco $(63,8 \%)$ cirurgias foram classificadas como potencialmente contaminadas, 32 $(31,3 \%)$ infectadas, $5(4,9 \%)$ contaminadas. Em quarenta e quatro $(43,1 \%)$ cirurgias foi feito o uso de antibioticoprofilaxia, $57(55,9 \%)$ tiveram terapia e $1(1 \%)$ não usou antibiótico. Em $14(13,7 \%)$ não se evidenciou uma justificativa para antibioticoterapia.

O índice de infecção do sítio operatório superficial (ISOS) foi de $26,7 \%$, sendo maior nas cirurgias consideradas potencialmente contaminadas (Tabela 5).

Oitenta e três $(81,4 \%)$ procedimentos foram realizados sob anestesia geral, $11(10,8 \%)$ subaracnóidea, 8 (7,8 \%) epidural. Não foram analisadas intercorrências anestésicas.

O tempo de cirurgia variou de 20 minutos até 8 horas, mostrando uma média de tempo de 2,85 horas por cirurgia.
Quarenta e oito $(47,1 \%)$ cirurgias tiveram complicação cirúrgica, sendo que $33(32,4 \%)$ tiveram uma complicação, 13 (12,7 \%) tiveram 2 complicações e 2 (2\%) tiveram 3 complicações.

A complicação mais temida nas cirurgias com anastomose $(\mathrm{n}=55)$ é deiscência, que foi evidenciada em 7(6,9\%) dos casos - tabela (6).

Ao analisar o índice de deiscência por tipo de sutura, observa-se que 6 pacientes de 23 anastomoses mecânicas apresentaram deiscência, enquanto que apenas $1(3,1 \%)$ de 32 anastomoses manuais apresentou essa complicação - tabela (7).

Foram realizadas $17(16,7 \%)$ ileostomias e 13 $(12,7 \%)$ colostomias.

Registro de óbito ocorreu em 11 (15,7\%) pacientes, cujos motivos foram: 3 (2,9\%) fístulas com sepse abdominal, 2 (2,0 \%) obstrução intestinal, $1(1,0 \%)$ perfuração de cólon pós-cirúrgica, $1(1,0 \%)$ perfuração de cólon após colonoscopia, $1(1,0 \%)$ DDC perfurada, $1(1,0 \%)$ gangrena sinérgica, $1(1,0 \%)$ pneumonia. Quatro (3,9\%) eram ASA III, 4 (3,9\%) eram ASA II e $3(2,9 \%)$ eram ASA I.

O tempo de internação dos pacientes variou de 1 dia até 82 dias, mostrando uma média de 25 dias de internação para cada paciente.

O seguimento dos pacientes teve uma média de 4,6 meses, sendo o máximo de 28,6 meses e o mínimo de 1 dia.

\section{DISCUSSÃO}

A SBCP exige do médico residente que sejam realizadas, ao final da formação, 72 cirurgias de gran-

Tabela 4 - Índice de deiscência e infecção de ferida operatória X preparo intestinal.

\begin{tabular}{|c|c|c|}
\hline & Com preparo $(\mathrm{N}=66 / 64,3 \%)$ & Sem preparo $(\mathrm{N}=36 / 35,3 \%)$ \\
\hline Deiscência & 6 pacientes & 1 paciente \\
\hline Infecção da FO & 22 pacientes $\quad(32,8 \%)$ & 5 pacientes $(14,3 \%)$ \\
\hline
\end{tabular}

Tabela 5 - Classificação cirúrgica quanto ao risco de infecção.

\begin{tabular}{lc}
\hline Classificação cirúrgica qto risco de infecção & Infecção do sítio operatório superficial (ISOS) \\
\hline Potencialmente contaminadas & $22(33,8 \%)$ \\
Cirurgias contaminadas & $1(20 \%)$ \\
Cirurgias infectadas & $4(12,5 \%)$ \\
\hline
\end{tabular}


Cirurgias Colorretais no Hospital Universitário da Universidade Federal de Sergipe: Três Anos da Criação do Serviço de Coloproctologia (Série Histórica) Juvenal da Rocha Torres Neto e Cols. de porte em 2 anos ${ }^{(2)}$. Essa padronização considera a mínima necessidade para a qualificação do ensino médico. O SC-HU/UFS obteve um número razoável de casos considerando o período de 30 meses. Foram catalogadas cerca de 40 cirurgias colorretais/ano, com média de 3,4 cirurgias/mês, totalizando cerca de 82 procedimentos de grande porte até o final da formação do médico residente. Durante o tempo estudado, houve apenas um médico residente participando das atividades do serviço, sendo para ele uma atuação satisfatória. Hoje são disponibilizadas duas vagas para residência médica em colo-proctologia no SC-HU/UFS, mantendo um crescente número de intervenções/ano, uma vez que esses números refletem o mínimo exigido nas normas da SBCP para instalação do programa de especialização médica ${ }^{(2)}$.

O serviço dispõe de cerca de 5 leitos inseridos num total de 28, que são redistribuídos para outras especialidades. São 2 salas cirúrgicas de grande porte, com 3 turnos reservados para rotina do serviço. Há perspectivas de melhora na catalogação dos pacientes com mínimo de perdas amostrais, já que o SC-HU/ UFS encontra-se em processo de informatização. Isto irá facilitar a pesquisa em futuros trabalhos.

As múltiplas cirurgias são importante fator no aumento da morbi-mortalidade e o tempo de internação ${ }^{(3)}$. MORRIS et al., demonstraram que a necessidade de reoperações de 5,8\% dos pacientes,

Tabela 6 - Complicações cirúrgicas.

\section{Complicação cirúrgica}

Complicações da ferida operatória

$32(31,4 \%)$

Abscessos abdominais

Fístulas

Deiscências de anastomose

Estenoses de anastomose

Sangramento da bolsa ileal

$13(12,7 \%)$

$11(10,8 \%)$

$7 \quad(6,9 \%)$

$2(2,0 \%)$

Obstrução intestinal

$1(1,0 \%)$

$1(1,0 \%)$ implicou num aumento da mortalidade precoce $(\mathrm{RR}=$ $2,4)$ e tempo de hospitalização $(\mathrm{RR}=2,2)^{(3)}$. O índice de reoperações do SC-HU/UFS foi de 22,9\% com uma mortalidade pós-operatória de $10,8 \%$, e média do tempo de internação de 25 dias, corroborando a necessidade de diminuir as reintervenções com conseqüente melhora das taxas morbi-mortalidade e otimização dos serviços prestados.

Atualmente, discute-se sobre o preparo de cólon como item imprescindível à realização de cirurgias colo-retais, inclusive com relatos de que o conteúdo líquido residual do preparo de cólon poderia ser igual ou pior que o não preparo de cólon. Apesar disso, a maioria dos serviços de colo-proctologia continua a utilizar rotineiramente preparo de cólon. Foi registrado o uso do manitol a $10 \%$ e fleet enema em $70 \%$ dos pacientes operados eletivamente. Apenas não foram preparados os pacientes submetidos à cirurgia de urgência. Autores relatam não haver maior índice de complicação em procedimentos realizados em cólons não preparados ${ }^{(3,4)}$. A presente série demonstra maior taxa de deiscência (9\%) em pacientes com preparo de cólon. Em pesquisas apoiadas pelo PIBIC desenvolvidas no Serviço de Cirurgia Experimental do HU/UFS em coelhos, encontrou-se índices semelhantes de complicações nas anastomoses com e sem preparo intestinal ${ }^{(5,6)}$. Estudos histomorfométricos das anastomoses colônicas complementarão dados do primeiro trabalho, avaliando outros fatores que poderiam contribuir para a deiscência de anastomose ${ }^{(6)}$. Contudo, é rotina do serviço o preparo de cólon em cirurgias eletivas e, quando oportuno, a realização de preparo colônico trans-operatório.

A profilaxia contra TVP/TEP foi aplicada em $80,4 \%$ dos casos, segundo diversos protocolos de profilaxia de TVP/TEP ${ }^{(7)}$.

As cirurgias sobre o cólon são consideradas, no mínimo, potencialmente contaminadas, sendo imprescindível a antibioticoprofilaxia, principalmente em grupos mais susceptíveis à infecção de sítio operatório superficial ${ }^{(8,9)}$. Quase a totalidade de pacientes usou antibiótico sob forma profilática ou terapêutica, con-

Tabela 7 - Índice de deiscência X anastomoses mecânicas ou manuais.

\begin{tabular}{lccc}
\hline & Número de anastomoses & Índice de deiscência \\
\hline Anastomose mecânica & 23 & $(41,8 \%)$ & $6(26,1 \%)$ \\
Anastomose manual & 32 & $(58,2 \%)$ & $1 \quad(3,1 \%)$ \\
Total & 55 & $(100 \%)$ & $7(12,7 \%)$ \\
\hline
\end{tabular}


forme diretrizes das comissões de infeccção hospi$\operatorname{talar}^{(10)}$. Em um paciente não há prescrição de profilaxia antibiótica, certamente por falha no preenchimento do prontuário. Antibioticoterapia foi empregada em $14(13,7 \%)$ pacientes sem indícios de infecção evidente nos prontuários. A literatura adverte que o uso indiscriminado de antibiótico têm implicações sérias tanto para o paciente quanto para a flora hospitalar, devido seleção de flora/resistência bacteriana ${ }^{(11)}$. Observou-se na nossa amostra um índice de 31,4\% para infecções da ferida operatória, representando a principal complicação infecciosa pós-operatória. Espera-se que esse índice reduza para níveis de 11-17\% observados em outros serviços ${ }^{(10,12)}$.

A deiscência de anastomose é uma complicação bastante temida, representando a maior causa de aumento da morbi-mortalidade no pós-operatório de cirurgias colorretais ${ }^{(13)}$. O índice de deiscência de anastomose da série foi maior do que a literatura $(12,7$ $\%)^{(13,14)}$. Foram confeccionadas anastomoses manuais e mecânicas no serviço. Entretanto, as anastomoses realizadas com grampeadores apresentaram maior deiscência $(26,1 \%)$ quando comparadas às manuais $(3,1 \%)$, discordando de outras experiências ${ }^{(15,16)}$.

As ileostomias de proteção foram empregadas nas $2(3,6 \%)$ proctocolectomias restaurativas.

Doença neoplásica do cólon indicou a maioria das cirurgias, perfazendo um total de $26,3 \%$ dos casos, acompanhando incidência relativamente elevada no mundo ocidental ${ }^{(17)}$. A dosagem do antígeno carcinoembrionário (CEA) é validado como marcador prognóstico de câncer colorretal ${ }^{(17,18)}$. Dos 18 casos de processo mitótico do cólon, havia registro do CEA em 17 deles, no entanto, com dosagem única em 13 pacientes, perdendo seu valor de marcador no seguimento pós-operatório.

$\mathrm{Na}$ cirurgia do trauma envolvendo lesões colônicas, a opção técnica por uma colostomia e fechamento do coto distal do reto é ainda bastante utilizada em nosso meio. Assim, a reconstrução do trânsito intestinal é o segundo tempo cirúrgico, sendo realizada em 10 casos.

Doenças inflamatórias intestinais somaram cerca de $9 \%$ das indicações cirúrgicas. Sabe-se que em cerca de $30-40 \%$ dos pacientes portadores de colite ulcerativa e 70-80 \% dos casos de Doença de Crohn, irão necessitar de uma cirurgia ao longo da vida. Foram operados $6(5,8 \%)$ casos por doença de Crohn (doença fistulosa complexa/sepse abdominal) e 2, por RCUI com intratabilidade clínica.

O megacólon chagásico é uma doença tropical e prevalente no Brasil, apesar da redução dos casos na última década observada pelos estudos epidemiológicos. O estado de Sergipe é zona endêmica, especialmente o sul do estado. Aproximadamente 10 $\%$ dos nossos pacientes foram operados por serem portadores da forma intestinal da doença. Dentre as diversas cirurgias descritas, o serviço escolhe a técnica de retossigmoidectomia abdomino-perineal com abaixamento retro-retal associada à anastomose colorretal mecânica à Duhamel modificada à Lins-Neto. Dos 10 pacientes operados por megacólon chagásico, a sorologia foi vista em 3 , sendo 1 , negativo.

A maioria dos serviços relata um seguimento pós-operatório pequeno, principalmente reflexo das condições sócio-econômico-culturais do país, e principalmente no Nordeste. Porém, encontramos um índice de seguimento pós-operatório elevado refletindo um comprometimento do serviço com o paciente, e uma normatização de condutas compatível com o objetivo de formação do médico residente.

\section{CONCLUSÃO}

O total de cirurgias que obtivemos durante o período, foi cerca de 40 cirurgias/ano, média suficiente para o SC-HU/UFS ser aceito como serviço pela SBCP, satisfazendo as necessidades para o ensino de médicos residentes em coloproctologia.

Continuamos realizando preparo de cólon para os procedimentos eletivos colorretais.

A profilaxia contra TVP/TEP foi realizada dentro dos critérios já bem estabelecidos.

O uso de antibiótico tem sido criterioso, embora tenhamos encontrado falha no preenchimento de prontuário justificando sua utilização.

A infecção de sítio operatório, deiscência de anastomose e sepse abdominal continuam sendo as principais complicações pós-operatórias.

O índice de reoperações foi elevado, refletindo diretamente no aumento da morbi-mortalidade e tempo de permanência hospitalar.

O rigor no preenchimento dos prontuários foi considerado bom, e as falhas anotadas para futuras correções.

A partir desses dados, os protocolos serão revistos e as condutas otimizadas. 
Rev bras Coloproct Janeiro/Março, 2008
Cirurgias Colorretais no Hospital Universitário da Universidade Federal de Sergipe: Três Anos da Criação do Serviço de Coloproctologia (Série Histórica) Juvenal da Rocha Torres Neto e Cols.
Vol. 28

\begin{abstract}
We studied colorectal surgeries carried through by the SC-HU/UFS from January 2004 to July 2006, historical series, referring to the creation of the colorectal medical residence. Registres by seventy patients that 53(75.7\%) were masculine sort and $\mathbf{1 7}(\mathbf{2 4 . 3 \%})$ feminine; medium age is 52 years. They had been submitted to the 102 procedures, 1,4 surgeries/patients, 29 reoperations. Nineteen $(18.6 \%)$ had been reconstitutions of intestinal transit, $15(14.8 \%)$ retossigmoidectomy, $11(10.8 \%)$ total colectomy, $9(8.8 \%)$ right colectomy, $6(5.8 \%)$ abdomino-perineais amputations, 3(2.9\%) proctocolectomy, $2(1.9 \%)$ left colectomy. Eighteen (17.6\%) surgeries were indicated for colorectal cancer, $8(7.8 \%)$ of rectum and $1(0.9 \%)$ of the anal canal; $10(9.9 \%)$ for megacólon; 10(9.9\%) previous colostomy, 5(5.9\%) Crohn's Disease, 5(4.9\%) DDC, 3(2.9\%) RCUI. Forty-eight (47.1\%) surgeries have had surgical complication: 32(31.4\%) ISOS, 13(12.7\%) abdominal abscess, 11(10.8\%) fístulas, 7(6.9\%) dehiscences of anastomoses, etc. We observe wound infection in $27(26.5 \%)$ surgeries. Fifty-five $(53.9 \%)$ patients had been submitted to the anastomoses, $32(58.2 \%)$ manual and $23(41.8 \%)$ stapled ones. It had dehiscence of anastomoses in $7(12.7 \%)$ surgeries: $1(3.1 \%)$ dehiscence for manual anastomoses and $6(26.1 \%)$ dehiscences for stapled anastomoses. Death in $11(15.7 \%)$ patient ones occurred. We evaluate the main data of the work objectifying to define goals, to elaborate and to improve the effective protocols, necessary to the good performance of the residence service.
\end{abstract}

Key Words: Colorectal surgery, Postoperative Complications, Colorectal Cancer, Ulcerative Colitis, Chagas'Disease.

\section{REFERÊNCIAS}

1. JENICEK, M. Towards evidence-based critical thinking medicine? Uses of best evidence in flawless argumentations. Med Sci Monit 2006; 12(8): RA 149-53.

2. SOCIEDADE BRASILEIRA DE COLOPROCTOLOGIA. Proposta para regulamentação dos programas de formação de especialistas em Coloproctologia. Disponível em http:// www.sbcp.org.br/residenciasbcp.htm. Acesso em 21/jan/07.

3. MORRIS, AM; BALDWIN, LM; MATTHEWS, B; DOMINITZ, JA; BARLOW, WE; DOBIE, SA. et al. Reoperation as a Quality Indicator in Colorectal Surgery: A Population-Based Analysis. Ann Surg. 2007 Jan; 245(1): 73-79.

4. NELSON, RL; DOLLEAR, T; FREELS, S; PERSKY, V. The Relation of Age, Race, and Gender to the Subsite Location of Colorectal Carcinoma. Cancer. 1997 Jul 15; 80(2): 193-7.

5. MENEZES, MVA. Comparação Entre Anastomose Primária de Cólon com e sem Preparo Intestinal em Coelhos. In: VI CONGRESSO DE INICIAÇÃO CIENTÍFICA-UFS/CNPQ, 2005, ARACAJU, 2005.

6. MENEZES, MVA. Estudo Histomorfométrico de Anastomoses Primárias de Cólon em Coelho com e sem Preparo Intestinal. In: XXIII CONGRESSO REGIONAL NORTE-NORDESTE DE COLOPROCTOLOGIA, 2006, FORTALEZA.

7. BERGQVIST, D. Venous Thromboembolism: a Review of Risk and Prevention in Colorectal Surgery Patients. Dis Colon Rectum. 2006 Oct; 49(10): 1620-8.

8. PLATELL, C; HALL JC The Prevention of Wound Infection in Patients Undergoing Colorectal Surgery. J Hosp Infect. 2001 Dec; 49(4): 233-8.

9. DE LALLA, F. Perioperative Antibiotic Prophylaxis: a Critical Review. Surg Infect (Larchmt). 2006; 7 Suppl 2: S37-9.

10. ROVERA, F; DIURNI, M; DIONIGI, G; BONI, L; FERRARI, A; CARCANO, G. et al. Antibiotic Prophylaxis in Colorectal Surgery. Expert Rev Anti Infect Ther. 2005 Oct; 3(5): 787-95.
11. MACHADO, A; BARROS, E; KONKEWICZ, LR. Antimicrobianos em Cirurgia. 1 ed. Porto Alegre: Artmed, 2006.

12. KONISHI, T; WATANABE, T; MORIKANE, K; FUKATSU, K; KITAYAMA, J; UMETANI, N. et al. Prospective Surveillance Effectively Reduced Rates of Surgical Site Infection Associated with Elective Colorectal Surgery at a University Hospital in Japan. Infect Control Hosp Epidemiol. 2006 May; 27(5): 526-8. Epub 2006 Apr 20.

13. KONISHI, T; WATANABE, T; KISHIMOTO, J; NAGAWA, H. Risk Factors for Anastomotic Leakage After Surgery for Colorectal Cancer: Results of Prospective Surveillance. J Am Coll Surg. 2006 Mar; 202(3): 439-44. Epub 2006 Jan 4.

14. WAGNER, OJ; EGGER, B. Influential Factors in Anastomosis Healing. Swiss Surg. 2003; 9(3): 105-13.

15. CHAMBERS, WM; MORTENSEN, NJ. Postoperative Leakage and Abscess Formation After Colorectal Surgery. Best Pract Res Clin Gastroenterol. 2004 Oct; 18(5): 865-80.

16. PLATELL, C; BARWOOD, N; DORFMANN, G; MAKIN, G. The Incidence of Anastomotic Leaks in Patients Undergoing Colorectal Surgery. Colorectal Dis. 2007 Jan; 9(1): 71-9.

17. CHENG, X; CHEN, VW; STEELE, B; RUIZ, B; FULTON, J; LIU, L. Subsite-specific Incidence Rate and Stage of Disease in Colorectal Cancer by Race, Gender, and Age Group in the United States 1992-1997. Cancer. 2001 Nov 15; 92(10): 2547-54.

18. FAKIH, MG; PADMANABHAN, A. CEA Monitoring in Colorectal Cancer. What You Should Know. Oncology (Williston Park). 2006 May; 20(6):579-87; discussion 588, 594, 596 passim.

Endereço para correspondência: JUVENAL DA ROCHA TORRES NETO

Endereço: Rua Ananias Azevedo, 100

Ed Piazza Fiorentina, apto. 902

Treze de Julho, Aracaju - SE - Brasil

Fax: (79) $3214-4830$

E-mail: jtorres@infonet.com.br 